\title{
Aux racines de la culture de guerre. Livres patriotiques pour enfants dans le Reich allemand durant l'immédiat avant- guerre
}

\author{
Bérénice Zunino \\ Institut français d'histoire en Allemagne, Francfort-sur-le-Main
}

Que ce serait bien si d'aventure une guerre avait éclaté et qu'un escadron de soldats défilait dans la rue [...]. Ah, mais toutes ces choses ne se trouvaient que dans les livres d'images, jamais on ne les voyait en vrai ; peut-être n'existaient-elles même pas ${ }^{1}$.

Ainsi s'exclame le petit Pierre Veraguth lors d'une de ses promenades solitaires dans le jardin familial. Si cet extrait du roman de Hermann Hesse, Roßhalde, écrit en 1912-1913, esquisse l'ennui ressenti dans la société allemande d'avant-guerre et l'espoir de renouveau incarné par un possible conflit futur, il renvoie aussi à la question de la mobilisation culturelle 2 . Ce phénomène qui opère comme tout processus sur le moyen ou long terme implique de sortir du strict cadre chronologique de la Première Guerre mondiale pour prendre en compte l'avant-guerre. Actuellement, celui-ci fait l'objet d'une attention accrue de la part des historiens ${ }^{3}$.

Nous nous proposons d'appréhender la veille de la guerre en analysant les racines de la culture de guerre enfantine allemande à partir de l'exemple de la littérature illustrée extrascolaire pour enfants entre 1911 et 1914 . La notion de culture de guerre, qui correspond à "un corpus de représentations du conflit cristallisé en un véritable système donnant à la guerre sa signification profonde $»^{4}$, mérite d'être envisagée dans une perspective diachronique. Peu reçue en Allemagne, elle a suscité de vifs débats dans le paysage historiographique français ${ }^{5}$; outre la question de savoir si elle est un facteur susceptible d'expliquer la ténacité des civils et/ou des soldats entre 1914 et 1918, se pose celle de sa formation: Annette Becker et Stéphane Audoin-Rouzeau présentent la culture de guerre comme une culture qui se serait cristallisée au début du conflit. Cette thèse se retrouve dans La guerre des enfants ${ }^{6}$ : les plus jeunes, qui représentent un enjeu particulier en période de guerre dans la mesure où ils incarnent l'avenir de la nation, auraient été impliqués dans le conflit dès les premières semaines d'août 1914. Or Nicolas Offenstadt n'a pas manqué de pointer les questions méthodologiques que

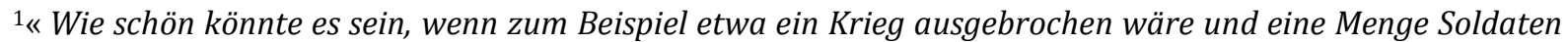
zu Pferde auf der Straße herankämen [...]. Ach, diese Sachen standen alle nur in den Bilderbüchern, in Wirklichkeit bekam man sie nie vor Augen, und vielleicht gab es sie gar nicht. » Hesse, Hermann, Roßhalde, Francfort/Main, Suhrkamp, 2015 (1re éd. 1914), p. 98. La traduction est le fait de l'auteure.

${ }^{2}$ Cette notion désigne l'ensemble des représentations collectives, des systèmes de valeurs et de croyances et des mesures institutionnelles prises par les États et les sociétés civiles. Horne, John, «Introduction: Mobilizing for "Total War", 1914-1918 », dans id. (dir.), State, Society and Mobilization in Europe during the First World War, Cambridge, Cambridge University Press, 1997, p. 1-17, en particulier p. 1.

${ }^{3}$ Voir le bilan du colloque international Guerres futures, guerres imaginées, organisé à Péronne en novembre 2011 : Jones, Heather, Weinrich, Arndt, « The pre-1914 Period : Imagined Wars, Future Wars Introduction », Francia. Forschungen zur westeuropäischen Geschichte, 2013, n 40, p. 305-315.

${ }^{4}$ Audoin-Rouzeau, Stéphane, Becker, Annette, 14-18, retrouver la Guerre, Paris, Gallimard, 2000, p. 145.

${ }^{5}$ Pour un bilan des débats entre les historiens de Péronne et ceux du CRID (Collectif de recherche internationale et de débats sur la guerre de 1914-1918) : Bauerkämper, Arnd, Julien, Élise, « Einleitung : Durchhalten! Kriegskulturen und Handlungspraktiken im Ersten Weltkrieg », dans id. (dir.) Durchhalten! Krieg und Gesellschaft im Vergleich 1914-1918, Göttingen, Vandenhoeck \& Ruprecht, 2010, p. 7-28.

${ }^{6}$ Audoin-Rouzeau, Stéphane, La Guerre des enfants, 1914-1918, 2éd., Paris, Colin, 2004, p. 51.
} 
soulève cette notion : une culture est un phénomène qui se forme sur le temps long7. Cette critique pose donc la question de la préexistence de la culture de guerre au conflit. Dans une perspective d'histoire culturelle qui accorde un intérêt particulier " à l'histoire des sens et des perceptions $»^{8}$, nous étudierons les formes visuelles de la littérature enfantine d'avant-guerre en prenant en compte les sensibilités des éditeurs. Les images, a fortiori lorsqu'elles s'adressent à des enfants dès l'âge de six ans peu rompus à la lecture de textes longs, participent au conditionnement de la mémoire culturelle ${ }^{9}$. Elles ne peuvent être analysées que replacées dans leur contexte (production, arrière-plan artistique, société du moment; difficile à évaluer, la diffusion fera ici seulement l'objet de quelques hypothèses). Plutôt que d'appréhender les évolutions quantitatives des sujets bellicistes pour la production entière de littérature enfantine, nous nous focaliserons sur des éditeurs sélectionnés pour leur diversité : leurs différences mettent en évidence des contrastes fructueux qui permettent de questionner la représentativité de la littérature patriotique ${ }^{10}$.

Cette approche amène à envisager l'avant-guerre sous un jour nouveau. L'analyse de cette « veille de guerre » n'a toutefois pas pour objectif d'écrire une histoire téléologique de l'avant-guerre; la Grande Guerre n'était pas inéluctable ${ }^{11}$. La "suggestion de la guerre» représente néanmoins l'une des caractéristiques de la "culture d'avantguerre »12. L'idée d'un conflit futur se fait de plus en plus présente dès 1911 : cette année marque la fin d'une phase de détente entre les grandes puissances (crise d'Agadir, guerre italo-turque en Lybie, guerres balkaniques) ainsi que l'intensification de l'encadrement de la jeunesse soutenu par l'État et l'armée. Deux ans plus tard, le centenaire de la bataille des Nations de 1813 achève de renforcer l'effervescence patriotique et contribue à une hausse des titres guerriers.

Nous commencerons par analyser le rôle de la peinture historique dans la transmission d'un imaginaire héroïque de la guerre aux enfants; ce genre est omniprésent dans l'ensemble du « bain visuel » ${ }^{13}$. Puis nous verrons que le boom patriotique provoqué par le centenaire des guerres napoléoniennes renforce les représentations idéalisées de la guerre. Enfin, nous relativiserons l'importance des thèmes guerriers avant 1914 en étudiant les disparités quantitatives et qualitatives selon les éditeurs.

\footnotetext{
${ }^{7}$ Offenstadt, Nicolas, « La Grande Guerre », dans C. Delacroix, F. Dosse, P. Garcia, id. (dir.), Historiographies. Concepts et débats, Paris, Gallimard, 2010, vol. 2, p. 1062-1073; p. 1068.

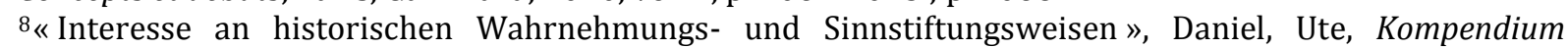
Kulturgeschichte. Theorien, Praxis, Schlüsselwörter, 5e éd., Francfort/Main, Suhrkamp, 2006, p. 12.

${ }^{9}$ Paul, Gerhard, «Das Jahrhundert der Bilder. Die visuelle Geschichte und der Bildkanon des kulturellen Gedächtnisses », dans id. (dir.), Das Jahrhundert der Bilder, vol. 1 : 1900 bis 1949, Göttingen, Vandenhoeck \& Ruprecht, 2009, p. 14-39.

${ }^{10}$ Olivera, Philippe, «Culture en guerre, culture d'exception ? Essai de mesure des formes de l'imprimé du temps de guerre», dans R. Cazals, E. Picard, D. Rolland (dir.), La Grande Guerre. Pratiques et expériences, Toulouse, Éditions Privat, 2005, p. 189-199. Nous nous permettons par ailleurs de renvoyer à la thèse suivante : Zunino, Bérénice, La littérature illustrée pour enfants à l'époque de la Première Guerre mondiale. Origines et évolution de la culture de guerre enfantine allemande, thèse de doctorat préparée sous la dir. de Jean-Paul Cahn (université Paris-Sorbonne) et Oliver Janz (Freie Universität Berlin), soutenue en 2014.

${ }^{11}$ Afflerbach, Holger, « The Topos of Improbable War in Europe before 1914 », dans id., D. Stevenson (dir.), An Improbable War? The Outbreak of World War I and European Political Culture before 1914, New York, Berghahn, 2007, p. 161-182.

${ }^{12}$ Alexandre, Philippe, Marcowitz, Reiner, «Introduction », dans id. (dir.), L'Allemagne en 1913 : culture mémorielle et culture d'avant-guerre. Deutschland im Jahre 1913 : Erinnerungs- und Vorkriegskultur, Nancy, Presses Universitaires de Nancy - Éditions Universitaires de Lorraine, 2013, p. 5-51 ; p. 12.

${ }^{13}$ Delporte, Christian, « De la légitimation à l'affirmation », dans id., L. Gervereau, D. Maréchal (dir.), Quelle est la place des images en histoire?, Paris, Nouveau Monde, 2008, p. 7-12 ; p. 12.
} 


\section{La peinture historique, fondement de la culture mémorielle à l'adresse des enfants}

Régis par un usage fonctionnel de l'histoire, les livres et illustrés font partie intégrante de la culture mémorielle du Kaiserreich ${ }^{14}$; conformément à l'opinion des élites militaires, ils véhiculent l'idée selon laquelle, pour assurer la pérennité de la nation, la guerre peut être utilisée comme un instrument politique légitime ${ }^{15}$. Cette conviction était répandue dans les milieux bourgeois, dont les enfants représentaient probablement, pour des raisons de pouvoir d'achat et de socialisation, le lectorat principal de la littérature illustrée.

Genre académique et national par excellence, la peinture historique, qui fait partie de la culture officielle depuis le XIX ${ }^{\mathrm{e}}$ siècle, véhicule une image apologétique et héroïque de la guerre. Omniprésente dans les livres et illustrés pour enfants, elle est appréciée par les théoriciens de l'art et les pédagogues pour ses vertus éducatives ${ }^{16}$; dans ces représentations de batailles, "la matière du récit est ordonnée, de manière à demeurer la plus pédagogique possible ${ }^{17}$. " Cette iconographie est d'autant plus propice à la transmission d'un engouement patriotique qu'elle se caractérise par un fort potentiel émotionnel ${ }^{18}$. Cette sensibilisation artistique vise à renforcer la fierté des jeunes Allemands en leur culture nationale, ainsi que leur sentiment d'appartenance à la même nation.

Assumant une fonction stabilisatrice, la littérature patriotique pour enfants est illustrée par des représentants de cette culture conservatrice. Ces peintres d'histoire et uniformologues étaient proches des cercles militaires et du pouvoir monarchique : outre Angelo Jank, Carl Becker, Hans Bohrdt, Anton Hoffmann, Franz Müller-Münster, Willy Stöwer ont collaboré avec des éditeurs de livres et illustrés pour enfants. Richard Knötel ${ }^{19}$ est certainement l'exemple le plus parlant de ces illustrateurs au service de l'ordre établi. Ce peintre officiel du Reich était familier de l'univers et du protocole militaires ; spécialisé dans l'uniformologie prussienne, il cofonda en 1898 la Société de science auxiliaire de l'histoire militaire (Gesellschaft für Heereskunde). Outre les toiles de batailles que lui commanda Guillaume II, il illustra dès la fin du XIX ${ }^{\mathrm{e}}$ siècle des albums ${ }^{20}$, des revues familiales conservatrices telles que Daheim et Über Land und Meer ainsi que

\footnotetext{
${ }^{14}$ Cornelißen, Christoph, «Erinnerungskulturen », dans F. Bösch, J. Danyel (dir.), Zeitgeschichte - Konzepte und Methoden, Göttingen, Vandenhoeck \& Ruprecht, 2012, p. 166-184.

${ }^{15}$ Mommsen, Wolfgang, « Der Topos vom unvermeidlichen Krieg. Außenpolitik und öffentliche Meinung im Deutschen Reich im letzten Jahrzehnt vor 1914 », dans J. Dülffer, K. Holl (dir.), Bereit zum Krieg. Kriegsmentalität im wilhelminischen Deutschland 1890-1914, Göttingen, Vandenhoeck \& Ruprecht, 1986, p. 194-224.

16Büttner, Frank, « Bildung des Volkes durch Geschichte. Zu den Anfängen öffentlicher Geschichtsmalerei in Deutschland», dans E. Mai (dir.), Historienmalerei in Europa. Paradigmen in Form, Funktion und Ideologie, Mayence, Verlag Philipp von Zabern, 1990, p. 77-94.

${ }^{17}$ Chaline, Olivier, «La bataille comme objet d'histoire», Francia. Forschungen zur westeuropäischen Geschichte, 2005, n² 2, p. 1-14 ; p. 3.

${ }^{18}$ François, Étienne, Siegrist, Hannes, Vogel, Jakob, «Die Nation. Vorstellungen, Inszenierungen, Emotionen », dans id. (dir.), Nation und Emotion. Deutschland und Frankreich im Vergleich 19. und 20. Jahrhundert, Göttingen, Vandenhoeck \& Ruprecht, 1995, p. 13-35.

19 « Knötel, Richard», dans U. Thieme, F. Becker (dir.), Allgemeines Lexikon der bildenden Künstler von der Antike bis zur Gegenwart, vol. 21, Leipzig, Seemann, p. 20-21.

${ }^{20}$ À titre d'exemple : Knötel, Richard, Röchling, Carl, Der alte Fritz in fünfzig Bildern für Jung und Alt, Berlin, Kittel, [1895]. Voir aussi pour la période étudiée : Knötel, Richard, Deutschlands Wehr zu Land und Meer, Hanovre, Molling \& Comp., [1912] ; id., Die eiserne Zeit vor hundert Jahren 1806-1813, Kattowitz, Carl Siwinna, Phönix-Verlag, [ca. 1913].
} 
des vignettes à collectionner de la marque Liebig et du chocolatier Ludwig Stollwerck ${ }^{21}$. Malgré sa mort en avril 1914, ses illustrations sont rééditées au début du conflit ${ }^{22}$. Les images apologétiques de la guerre véhiculées dans la littérature enfantine à la veille de 1914 sont donc réalisées par des artistes au profil particulier.

La littérature patriotique pour enfants s'insère par ailleurs dans un vaste bain visuel qui accorde une place importante au culte des chefs militaires et à la loyauté envers la monarchie. Les écoliers étaient familiers des œuvres de commande qui étaient reproduites dans de nombreux supports populaires et occupaient une place importante dans les écoles, ornées de statues de personnalités, telles que Guillaume II et l'impératrice Auguste Victoria, ainsi que de tableaux de peinture historique ${ }^{23}$. Ces toiles étaient même accrochées aux murs des salles de classe sous forme d'affiches ${ }^{24}$. Les enfants côtoyaient ces formes iconographiques dans les musées, comme la Zeughaus de Berlin, et dans l'architecture urbaine, dont l'allée de la victoire symbolisait l'hégémonie prussienne ${ }^{25}$; ils y étaient confrontés par le biais du panorama, florissant depuis le dernier tiers du XIX siècle, et de l'imagerie populaire à tendance patriotique qui décorait les intérieurs bourgeois. Les palimpsestes présents dans la littérature illustrée reposent donc sur un jeu de connivence avec les jeunes lecteurs tout en stimulant leur mémoire.

Médium privilégié de l'éducation patriotique et esthétique des jeunes générations, la littérature patriotique pour enfants est aussi l'expression d'un "militarisme folklorique $»^{26}$ : révélateurs de l'importance des valeurs militaires, ces livres sont des objets de divertissement pour les enfants tout en représentant un débouché commercial. Les éditeurs s'attachent à développer des stratégies commerciales en misant sur le discours idéologique et la culture mémorielle du Kaiserreich ; par exemple, les dédicaces à des membres des familles princières et impériale placées en tête d'ouvrages leur permettent d'afficher leur loyauté envers la monarchie : Deutschland in Waffen est par exemple "dédié à Sa Majesté l'Empereur et le Roi » ${ }^{27}$. Assez fréquentes, elles ne se limitent pas aux livres patriotiques et sont considérées comme un signe de bonne qualité28.

Dans cette logique commerciale, les éditeurs saisissent l'opportunité des festivités de 1913 pour exploiter cette culture mémorielle. Ils recourent de nouveau à la tradition iconographique de la peinture d'histoire héroïque, dont nous aimerions analyser un exemple représentatif dans la deuxième partie.

\footnotetext{
21 Jussen, Bernhard, «Liebigs Sammelbilder. Weltwissen und Geschichtsvorstellung im Reklamesammelbild», dans : G. Paul, op. cit., p. 132-139.

${ }^{22}$ Knötel, Richard, Fernau, Peter, Unsere Feldgrauen und Blaujacken, Hanovre, Molling \& Comp., [1915].

${ }^{23}$ Pust, Hans-Christian, "Vaterländische Erziehung" für "Höhere Mädchen". Soziale Herkunft und politische Erziehung von Schülerinnen an höheren Mädchenschulen in Schleswig-Holstein, 1861-1918, Osnabrück, Der Andere Verlag, 2004.

${ }^{24}$ R. Hemprich, R. Fritzsche (dir.), Ratgeber für deutsche Lehrer und Erzieher. Wissenschaftliches Sammelwerk für alle Unterrichtsfächer und zur Fortbildung, vol. 7 : Das Bilderbuch und Werke bildender Kunst im Unterricht, Langensalza, Julius Beltz, 1911.

${ }^{25}$ Piper, Ernst, « Das kulturelle Leben im Kaiserreich », dans B. Heidenreich, S. Neitzel (dir.), Das Deutsche Kaiserreich 1890-1914, Paderborn, Schöningh, 2011, p. 75-96.

${ }^{26}$ «Folkloremilitarismus », Vogel, Jakob, Nationen im Gleichschritt, Göttingen, Vandenhoeck \& Ruprecht, 1997, p. 275.

${ }^{27}$ «Seiner Majestät dem Kaiser und König», dans Deutschland in Waffen, Stuttgart / Berlin, Deutsche Verlags-Anstalt, [1913], p. 3.

${ }^{28}$ Ries, Hans, Illustration und Illustratoren des Kinder- und Jugendbuchs im deutschsprachigen Raum 18711914. Das Bildangebot der Wilhelminischen Zeit. Geschichte und Ästhetik der Original- und Drucktechniken. Internationales Lexikon der Illustratoren, Osnabrück, Wenner, 1992.
} 


\section{Le boom patriotique de 1913 : exemple représentatif de l'iconographie traditionnelle}

Comme le jubilé d'argent du règne de Guillaume II, en juin 1913, la commémoration du centenaire de la bataille des Nations, le 18 octobre 1813, a suscité en Allemagne une effervescence patriotique ${ }^{29}$. Outre les portraits de Guillaume II qui se multiplient, les guerres contre l'hégémonie napoléonienne sont l'un des principaux sujets qui font entrer plus largement le discours sur la guerre dans la littérature pour enfants. Ces deux événements mémoriels marquent une intensification du discours patriotique dans des revues telles que le Deutscher Kinderfreund ${ }^{30}$, dont la date de création assez ancienne (1878) donne à penser qu'elle a connu un succès certain auprès du jeune public. Outre la distribution de brochures sur les campagnes anti-napoléoniennes dans les écoles et lors des fêtes commémoratives, l'édition par les mouvements de jeunesse d'ouvrages spécialisés et la production de cartes postales à cette occasion ${ }^{31}$, des albums pour enfants sont entièrement consacrés à ce sujet. À l'occasion de la vague commémorative, la maison Scholz (Mayence) en fait sa spécialité : alors qu'elle n'a produit que deux albums patriotiques entre 1907 et 1910, elle lance en 1912 sa série d'ouvrages patriotiques Vaterländische Bilderbücher, également appelée Vaterländisches Bilderwerk ${ }^{32}$. Cette année-là, ces livres constituent $20 \%$ de sa production pour atteindre $25 \%$ l'année suivante 33 .

Les images, construites sur des références à des toiles de commande, y forment le cœur du récit. Le portrait équestre de l'archiduc Charles-Louis d'Autriche-Teschen durant la bataille d'Essling dans Zehn Jahre deutscher Not (1803-1812) en est un exemple représentatif.

\footnotetext{
${ }^{29}$ Siemann, Wolfgang, « Krieg und Frieden in historischen Gedenkfeiern des Jahres 1913 », dans D. Düding, P. Friedemann, P. Münch (dir.), Öffentliche Festkultur. Politische Feste in Deutschland vor der Aufklärung bis zum Ersten Weltkrieg, Hambourg, Rowohlt, 1988, p. 298-320.

${ }^{30}$ Deutscher Kinderfreund, Ninck, Hambourg, Expedition des Deutschen Kinderfreunds, 1913.

${ }^{31}$ Aslangul, Claire, «La Jugendbewegung et les images. Formes et fonctions de l'iconographie des mouvements de jeunes dans le premier tiers du XXe siècle », Recherches germaniques, 2009, Hors-série $\mathrm{n}^{\circ} 6$ : Mouvements de jeunesse et jeunes en mouvement, université de Strasbourg, p. 111-131.

${ }^{32}$ Markmann, Hans-Jochen, "Kriegspädagogik als Lebensaufgabe - Wilhelm Kottenrodt (1878-1948)", dans Arbeitsgruppe Lehrer und Krieg (dir.), Lehrer helfen siegen. Kriegspädagogik im Kaiserreich, Berlin, Diesterweg-Hochschule, 1987, p. 69-90.

${ }^{33}$ Schneider, Cornelia, Die Bilderbuchproduktion der Verlage Jos. Scholz (Mainz) und Schaffstein (Köln) in den Jahren 1899 bis 1932, thèse de l'université Goethe de Francfort-sur-le-Main, soutenue en 1984.
} 


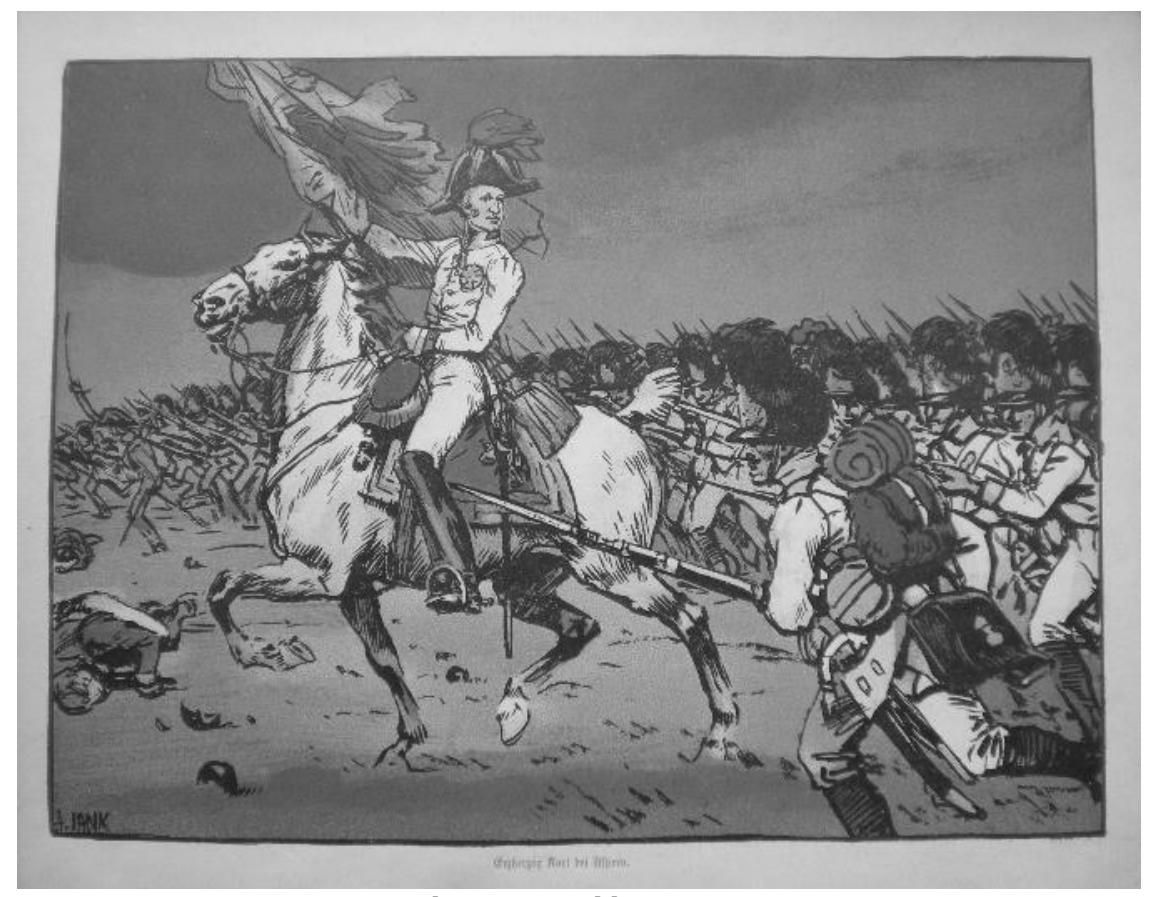

Erzherzog Karl bei Aspern.

Dans Jank, Angelo, Deutschlands Not und Befreiung, vol. 1 : Zehn Jahre deutscher Not (1803-1812),

Mayence, Scholz, [1912], p. 8.

(C) Staatsbibliothek zu Berlin, Kinder- und Jugendbuchabteilung, cote : B III b, 918.

Son illustrateur Angelo Jank, membre de la Sécession munichoise et représentant de l'Art nouveau, disposait d'une double formation artistique et militaire qu'il mit à profit d'une carrière d'uniformologue et de peintre historique ${ }^{34}$. L'image et le texte qui l'accompagne reprennent l'épisode, non attesté mais devenu légendaire, de l'archiduc Charles d'Autriche saisissant le drapeau de son régiment et défiant l'ennemi durant la bataille d'Essling, les 21 et 22 mai 1809. Vu de profil, Charles d'Autriche, tel un condottiere, domine la scène du haut de son lipizzan majestueux, dont la posture est calquée sur celle du même personnage représenté dans les célèbres toiles de Johann Peter Krafft L'archiduc Charles à la bataille d'Essling (1819) et Les vainqueurs d'Essling (1820). Inspirés de la peinture historique, ces éléments rhétoriques conventionnels de l'image soulignent la persévérance et le courage du héros guerrier ${ }^{35}$; le choix du moment déterminant de l'action renforce aussi le caractère héroïque de la scène ${ }^{36}$. Conformément aux ressorts du récit national, la victoire est mise en scène de manière idéalisée ${ }^{37}$, d'autant plus que la bataille d'Essling fut le prélude à la fin du mythe de l'invincibilité de Napoléon.

\footnotetext{
${ }^{34}$ Aichner, Ernst, «Bayerische Militärmaler. Ihre Werke als historische Primärquellen », dans cat. exp. Bayerische Militärmaler. Von Beich bis Thöny, Bayerisches Armeemuseum Ingolstadt, Ingolstadt, 1982, p. 7-26.

${ }^{35}$ Aslangul, Claire, Représentations de la guerre chez les peintres, graveurs et dessinateurs allemands au $X X^{e}$ siècle, dans le contexte européen : traditions, évolutions et ruptures dans les codes iconographiques, thèse de doctorat de l'École Pratique des Hautes Etudes et de la Technische Universität de Dresde, soutenue en 2003, p. 51.

${ }^{36}$ Werner, Elke Anna, "Schlachtenbild», dans U. Fleckner, M. Warnke, H. Ziegler (dir.), Handbuch der politischen Ikonographie, vol. 2, Munich, Beck, 2011, p. 332-340.

${ }^{37}$ François, Étienne, Schulze, Hagen, «Das emotionale Fundament der Nationen », dans M. Flacke (dir.), cat. exp. Mythen der Nationen. Ein europäisches Panorama, Berlin, Deutsches Historisches Museum, 1998, p. 17-32.
} 
La perspective adoptée correspond pourtant à un mythe autrichien ${ }^{38}$ : l'archiduc Charles, issu de la dynastie des Habsbourg contre laquelle les Hohenzollern se sont imposés en 1866 et dont la statue trône aujourd'hui encore sur la " place des Héros » devant la Hofburg, est proposé en modèle héroïque aux jeunes lecteurs allemands. L'aigle noir, susceptible de rappeler le symbole prussien puis allemand, visible dans l'illustration, mais absent de la toile de Krafft, renforce l'assimilation de l'Autriche à la Prusse. Celle-ci exprime sans doute les bonnes relations qu'entretiennent les deux États depuis la signature de la Duplice en 1879. Présentée du point de vue des Autrichiens, la scène permet, surtout, de passer sous silence l'affaiblissement de la Prusse par la paix de Tilsit, en 1807, et l'alliance conclue par la Bavière avec Napoléon. Elle donne ainsi l'illusion d'une résistance inébranlable à la France.

Cette iconographie au fort potentiel émotionnel ne sert pas, comme c'est parfois le cas durant les commémorations officielles du centenaire de 1813, de prétexte pour évoquer la guerre (sur deux fronts) qui menace, aux yeux des acteurs politiques et militaires, le Reich. En proposant aux jeunes générations une grille de lecture idéalisée du passé, elles ont pour fonction de réaffirmer la puissance du Reich et, indirectement, de sensibiliser les enfants à l'avenir de la nation. Les guerres anti-napoléoniennes sont d'autant plus susceptibles d'interpeller les jeunes lecteurs qu'elles constituent l'un des piliers de l'enseignement de l'histoire à l'école, a fortiori depuis les réformes scolaires de 1889.

Autrement dit, le traitement de ces guerres dans un style suranné et héroïque a contribué grandement au boom mémoriel et patriotique de 1913 par le biais de la littérature illustrée. Ces thèmes guerriers sont particulièrement exploités par des éditeurs qui affichent leurs affinités avec le pouvoir monarchique et militaire.

\section{Des éditeurs proches des cercles conservateurs et nationalistes}

Tout en touchant largement le marché du livre pour enfants, le boom patriotique aux alentours de 1913 n'a pas affecté tous les éditeurs avec la même ampleur. La maison d'édition Schreiber (Esslingen), figure de proue en matière de livres d'images de guerre (Kriegsbilderbücher) entre 1914 et 1918, édite peu de livres patriotiques avant le début $\mathrm{du}$ conflit. Les thèmes guerriers font partie de son offre traditionnelle, mais ils sont restreints ${ }^{39}$.

A contrario, certains éditeurs qui exploitent particulièrement ces thèmes affichent leur proximité avec des cercles conservateurs. Le Phönix-Verlag/Carl Siwinna (Kattowitz), éditeur de l'album Die eiserne Zeit vor hundert Jahren $1806-1813^{40} \mathrm{et}$ de la brochure illustrée 1813-1913. Ein Festbuch für die deutsche Jugend41, aussi bien consacrée aux "guerres de libération» (selon le terme allemand de Befreiungskriege, 1813-1815) qu'au culte de Guillaume II, présente la particularité d'être fournisseur à la cour du roi et de l'empereur de Prusse (Hoflieferant des Königs und Kaisers von Preußen).

L'exemple de Scholz, réputé pour « l'orientation fortement nationale de son programme éditorial $»^{42}$, est le plus radical : en 1913, la part de sa production consacrée aux titres patriotiques (25\%) dépasse nettement la moyenne de $12 \%$ calculée sur la base des

\footnotetext{
38Bruckmüller, Ernst, « Österreich. "An Ehren und an Siegen reich” », dans ibidem, p. 269-294.

${ }^{39}$ Landesmuseum Württemberg (LMW), collection Schreiber « VK (Volkskunde) Populäre Druckgraphik Unterlagen zu einzelnen Produktionen - Bücher ».

${ }^{40}$ R. Knötel [ca. 1913], op. cit.

${ }^{41}$ Knötel, Paul, Wilhelm II, 1813-1913. Ein Festbuch für die deutsche Jugend, Kattowitz, Carl Siwinna / Phönix-Verlag, [1913]. (Paul Knötel était le frère de Richard Knötel).

42 « Die betont nationale Ausrichtung des Verlagsprogramms », C. Schneider, op. cit., p. 151.
} 
titres répertoriés dans le Kaysers Bücherverzeichnis ${ }^{43}$. De surcroît, les critiques positives dont les ouvrages de Scholz font l'objet dans les bibliographies spécialisées, ainsi que leur mention dans les catalogues de bibliothèques scolaires, donnent à penser qu'ils ont connu un certain succès ${ }^{44}$. En outre, la série précitée Vaterländische Bilderbücher est gérée par Wilhelm Kottenrodt, ancien maître d'école connu sous le pseudonyme de Wilhelm Kotzde pour son engagement nationaliste. Dans un pamphlet nationaliste qu'il a coédité à l'occasion du centenaire de 1813, il revendique la dimension idéologique et mobilisatrice des albums de sa série, en particulier des trois volumes de Deutschlands Not und Befreiung consacrés aux campagnes contre l'hégémonie napoléonienne :

Le peuple allemand se mobilise pour le jubilé des guerres de libération. Ce sont des souvenirs solennels qui pénètrent à présent les arrière-petits-enfants de cette génération héroïque et qui font sentir à notre jeunesse un souffle moral par lequel le dévouement fidèle à la Patrie jusque dans la mort apparaît comme une évidence. ${ }^{45}$

Selon Scholz et Kotzde, les enfants doivent se montrer dignes de leurs ancêtres en perpétuant leurs actes héroïques. Ces exhortations sont révélatrices des attentes envers les jeunes générations et de leur peur face à l'avenir. Elles alimentent aussi la «psychose de guerre ${ }^{46}$ de plus en plus palpable à la veille de 1914 ; les entreprises éditoriales de Scholz et Kotzde sont soutenues par des représentants militaires actifs dans des organisations nationalistes, dont le commandant Keim rattaché à la Ligue pour la Défense (Wehrverein), tout juste fondée fin 1912, et le commandant Mey, représentant de la section berlinoise de la Ligue pangermaniste (Alldeutscher Verband) ${ }^{47}$.

Une partie au moins des livres patriotiques d'avant 1914 est par conséquent révélatrice d'un phénomène plus restreint que le militarisme folklorique mais symptomatique de l'avant-guerre: certains d'entre eux sont l'expression d'un "militarisme de conviction ${ }^{48}$ et reflètent la radicalisation de la vie politique à partir de 1911-1912, dans le contexte de la crise d'Agadir et de la victoire des sociaux-démocrates au Parlement. Les éditeurs Siwinna et Scholz, proches du pouvoir monarchique pour l'un et des cercles nationalistes pour l'autre, se sont appropriés avec zèle les canons esthétiques traditionnels qui véhiculent un imaginaire héroïque de la guerre.

En août 1914, les populations n'ont pas accueilli les hostilités dans un élan d'enthousiasme général ${ }^{49}$. Toutefois, la littérature patriotique pour enfants, en tant que composante de l'éducation des jeunes générations, a probablement joué un rôle dans le

\footnotetext{
${ }^{43}$ Christadler, Marieluise, Kriegserziehung im Jugendbuch. Literarische Mobilmachung in Deutschland und Frankreich vor 1914, Francfort/Main, Haag/Herchen, 1978, p. 67.

${ }^{44}$ B. Zunino, op. cit.

${ }^{45}$ «Das deutsche Volk rüstet sich, die Jahrhundertfeier der Befreiungskriege zu begehen. Weihevolle Erinnerungen sind es, die heute die Urenkel jenes Heldengeschlechts durchdringen und namentlich unsere Jugend einen Hauch des Geistes spüren lassen, der die treue Hingebung fürs Vaterland bis in den Tod als etwas Selbstverständliches empfindet. » Kotzde, Wilhelm, Scholz, Joseph, Der Kampf um die Jugendschrift, Mayence, Scholz, 1913, p. 89.

46P. Alexandre, R. Marcowitz, op. cit., p. 15.

${ }^{47}$ Kotzde, Wilhelm, «Einige Spitzen des Deutschen Lehrervereins im Kampf um die Jugendschrift », dans id., Scholz, op. cit., p. 121-156.

${ }^{48}$ Rohkrämer, Thomas, "Der Gesinnungsmilitarismus der "kleinen Leute" im Deutschen Kaiserreich », dans W. Wette (dir.), Der Krieg des kleinen Mannes. Eine Militärgeschichte von unten, Munich, Piper, 1992, p. 95-109.

${ }^{49}$ Verhey, Jeffrey, Der « Geist von 1914 » und die Erfindung der Volksgemeinschaft, Hambourg, Hamburger Edition, 2000.
} 
conditionnement des esprits en contribuant à déréaliser la guerre et à " fabriquer » ${ }^{50}$ un engouement pour la défense de la nation, d'autant plus que la vague commémorative de 1913 en a favorisé la hausse. Typique du bain visuel de l'époque, la peinture historique représente le fondement de l'imaginaire héroïque dans les livres et illustrés pour enfants qui persiste après 1914. L'exploration de la littérature illustrée pour enfants à la veille de 1914 montre donc que la culture de guerre n'est pas apparue ex nihilo durant les premières semaines de la Grande Guerre; empreinte de canons esthétiques traditionnels, la culture mémorielle du Kaiserreich constitue un terrain favorable à son développement entre 1914 et 1918.

Eu égard aux disparités dans le paysage éditorial, la Première Guerre mondiale n'a pas représenté la même césure pour toutes les maisons d'édition. À la veille de la guerre, la littérature patriotique pour enfants, illustrée par des artistes proches des cercles militaires, est en partie produite par des éditeurs qui affichent leurs affinités avec des représentants de la monarchie ou des associations nationalistes. Après le début du conflit, de nombreux éditeurs auparavant peu versés dans le patriotisme accordent, probablement par intérêt économique, mais aussi en raison de la pression sociale, une part plus importante de leur programme éditorial à la production de livres de guerre. Tel est le cas de Schreiber. Catalyseur, la guerre a été un facteur de poursuite et d'amplification de la production patriotique pour certaines maisons d'édition, tout en marquant une rupture plus nette pour les autres.

\footnotetext{
${ }^{50}$ Cahn, Jean-Paul, Knopper, Françoise, Saint-Gille, Anne-Marie, «Introduction », dans id. (dir.), De la guerre juste à la paix juste. Aspects confessionnels de la construction de la paix dans l'espace franco-allemand (XVI ${ }^{e_{-}}$ $X X^{e}$ ), Villeneuve d'Ascq, Presses Universitaires du Septentrion, 2008, p. 7-17; p. 7.
} 